\title{
Craniovertebral Junction Instability: A Review of Facts about Facets
}

\author{
Atul Goel \\ Department of Neurosurgery, King Edward Memorial Hospital and Seth Gordhandas Sunderdas Medical College, Mumbai, India
}

Craniovertebral junction surgery involves an appropriate philosophical, biomechanical and anatomical understanding apart from high degree of technical skill and ability of controlling venous and arterial bleeding. The author presents his 30-year experience with treating complex craniovertebral junction instability related surgical issues. The facets of atlas and axis form the primary site of movements at the craniovertebral junction. All craniovertebral junction instability is essentially localized to the atlantoaxial facet joint. Direct manipulation and fixation of the facets forms the basis of treatment for instability.

Keywords: Atlantoaxial facet joint; Basilar invagination; Chiari malformation; Syringomyelia

\section{Introduction}

Craniovertebral junction is supremely designed and immaculately structured to perform its unique role of providing stability and mobility to the most stable and most mobile regions of the body. It also performs its role of protection of most critical neural and vascular structures whilst permitting a wide range of movements to great perfection.

The craniovertebral complex comprises of two sets of joints-occipitoatlantal and atlantoaxial joints. Whilst occipitoatlantal joint is the most stable, atlantoaxial is the most mobile of all joints of the body. The strength of ligaments associated with the occipitoatlantal joint can be appreciated only by direct inspection during cadaveric dissections. The ligaments are remarkably strong and 'steel-like' and it takes considerable effort to dis-lodge the joint. On the other hand, atlantoaxial joint is supple and is most susceptible to instability. Our study of cranioverte- bral junction of various animals demonstrated that more mobile the craniovertebral junction is, more unstable it is. The head of a bird can rotate 360 degrees, but twisting with a human hand can break the neck. On the other hand, the neck movements are limited to only 30 degree in a lion, but the junction is so strong that no human effort can dislodge the joint.

\section{Historical Perspectives}

Craniovertebral junction anomalies were only anatomical and radiological curiosities till not long ago. Improved imaging, understanding of anatomical subtleties and the possibility of evaluation of the region with advanced computer based imaging hallmarked a therapeutic revolution in the subject. The unfortunate patients harboring such anomalies and having neurological deficits related to affection of the region can now hope to live and survive with pride and comfort.

Received Apr 22, 2015; Accepted Apr 23, 2015

Corresponding author: Atul Goel

Department of Neurosurgery, King Edward Memorial Hospital and Seth Gordhandas Sunderdas Medical College,

Parel, Mumbai 400012, India

Tel: +91-22-2412-9884, Fax: +91-22-2414-3435, E-mail: atulgoel62@hotmail.com 
Till not long ago, the treatment of craniovertebral junction instability was divided into two groups on the basis of presence or absence of atlantoaxial instability. If the atlantoaxial region was considered to be mobile and reducible, atlantoaxial fixation was advocated. On the other hand, if the joint was considered to be 'fixed,' anterior transoral decompression or posterior foramen magnum decompression was advocated. The dictum of anterior surgery for an anterior compressive lesion and posterior surgery for posterior compression was established $[1,2]$. In basilar invagination, the atlantoaxial joint was considered to be 'fixed' or 'irreducible' and accordingly decompressive surgery was the mainstay of treatment. Transoral surgery became highly popular as a mode of decompression in the later years of 1980's. Some surgeons advocated posterior fixation following transoral decompression surgery as removal of odontoid process and adjoining bones was considered to have a de-stabilizing effect. For atlantoaxial dislocations that were reducible, midline wiring over the arch of atlas and lamina of $\mathrm{C} 2$ and bone placement and fixation formed the basis of stabilization procedures [3-5].

\section{Occipitocervical Fixation}

Inclusion of the occipital bone in the fixation constructs became popular in the 1980's. The implants included loops and contoured metal rods. The fixation of the occipital end of the implant was done earlier with the help of wires and subsequently with the assistance of screws. However, as the biomechanical issues of the region were understood, the focus of craniovertebral junction stabilization techniques is now only on segmental atlantoaxial fixation. It may not be incorrect to state that all craniovertebral instability is equivalent to atlantoaxial instability. Occipitoatlantal instability is seen only in extreme trauma or sometimes in pediatric age patients and is generally related to syndromic affection of multiple joints. Craniovertebral junction stabilization is essentially equivalent to atlantoaxial stabilization. Direct atlantoaxial fixation in cases with basilar invagination is relatively a difficult technical surgery. However, if it is done satisfactorily and completely, it provides a segmental stabilization at the point of fulcrum of all movements. Inclusion of occipital bone in the fixation construct provides a suboptimal form of fixation, as it involves inclusion of a normal joint in the fixation assembly. Although inclusion of the occipital bone in the fixation construct is still popular, the technique is gradually but surely receiving less favor.

\section{Atlantoaxial dislocation (Fig. 1)}

In 1986, Magerl introduced transarticular method of fixation [6]. The technique has remained remarkably popular due to its inherent biomechanical superiority, relative ease of conduct of surgery and satisfactory long-term clinical outcomes. In the year 1988, Goel and Laheri [7] introduced a technique of atlantoaxial fixation that involved individual insertion of screws into the facet of atlas and of axis [8]. The technique involved opening of the joint, denuding of articular cartilage and introduction of bone graft within the joint prior to fixation with plate/rod and mono-polyaxial screws. The relative safety of vertebral artery and the biomechanical strength of the technique have made it the most preferred technique of atlantoaxial fixation. The possibility and safety of sectioning of the large $\mathrm{C} 2$ ganglion located posterior to the joint for the purpose of implant fixation opened up a new chapter in the history of fixation of atlantoaxial joint [9]. Despite the fact that the sectioning of the $\mathrm{C} 2$ ganglion is safe, ganglionectomy should be avoided whenever it is possible. Superior and inferior mobilization of the ganglion can assist in appropriate exposure for screw insertion and placement of the implants. Facetal fixation now forms the basis of atlantoaxial fixation techniques.

\section{Atlantoaxial Facetal Fixation (Fig. 1)}

The facets are the only true joints of the spine in general and atlantoaxial region in particular [10]. Most movements in the region are initiated and completed in the atlantoaxial facet joint. Considering that the joint is extensively mobile in normal life, the possibility of instability is relatively a common feature. Atlantoaxial joint forms the fulcrum of all movements and stabilizing the joint presents the best opportunity for fixating and arthrodesis. Direct atlantoaxial fixation provides the biomechanically strongest method of stabilization. However, presence of a large venous plexus in the lateral gutter makes the conduct of surgery relatively difficult. Presence of basilar invagination, assimilation of atlas, short neck, bone fusions etc. signal rostral location of the atlantoaxial joint and the consequent difficulties in surgical exposure and instrumentation. If the atlantoaxial joint and the adjoining fac- 
ets are widely exposed by surgery, various permutations and combination of techniques can be possible. The site, trajectory and the mode of screw insertion into the facets can be altered as per the needs of an individual situation. The C2 screw can even be inserted in a transarticular method [11]. In situations with high-riding vertebral artery, the screw may be inserted medially and even into the body of C2 vertebra [12]. The technique provides a 'zeromovement' situation that is not possible with other techniques. Metal implants hold the region for few weeks, after which bone fusion has to occur to stabilize the region and to hold the implants. Bone placed into the articular cavity not only blocks the main site of segmental movements but also assists in stabilization of the implants and the joint. The additional bone surface available provides an enhanced space for bone fusion.

The facets of atlas and axis are located in the lateral gutter of the region. The region is loaded with a large plexus of veins. Venous bleeding can sometimes be quite significant and can adversely affect the conduct of the operation. Understanding of the bone anatomy in its three dimensional perspective is crucially important. The vertebral artery has multiple twists and turns especially in the craniovertebral junctional region. These loops have a dynamic relationship with the bones and grooves and participate in movements of head and neck [12]. The relationship of the vertebral artery to the facet of axis, arch of atlas and in the region lateral to the facet joint needs to be understood, appropriately evaluated and the artery needs to be precisely handled during the conduct of surgery. The course of the artery in relationship to the facet of $\mathrm{C} 2$, makes it vulnerable during screw implantation. Appropriate evaluation of the traverse of the artery on the basis of computer-based imaging can assist in providing safety
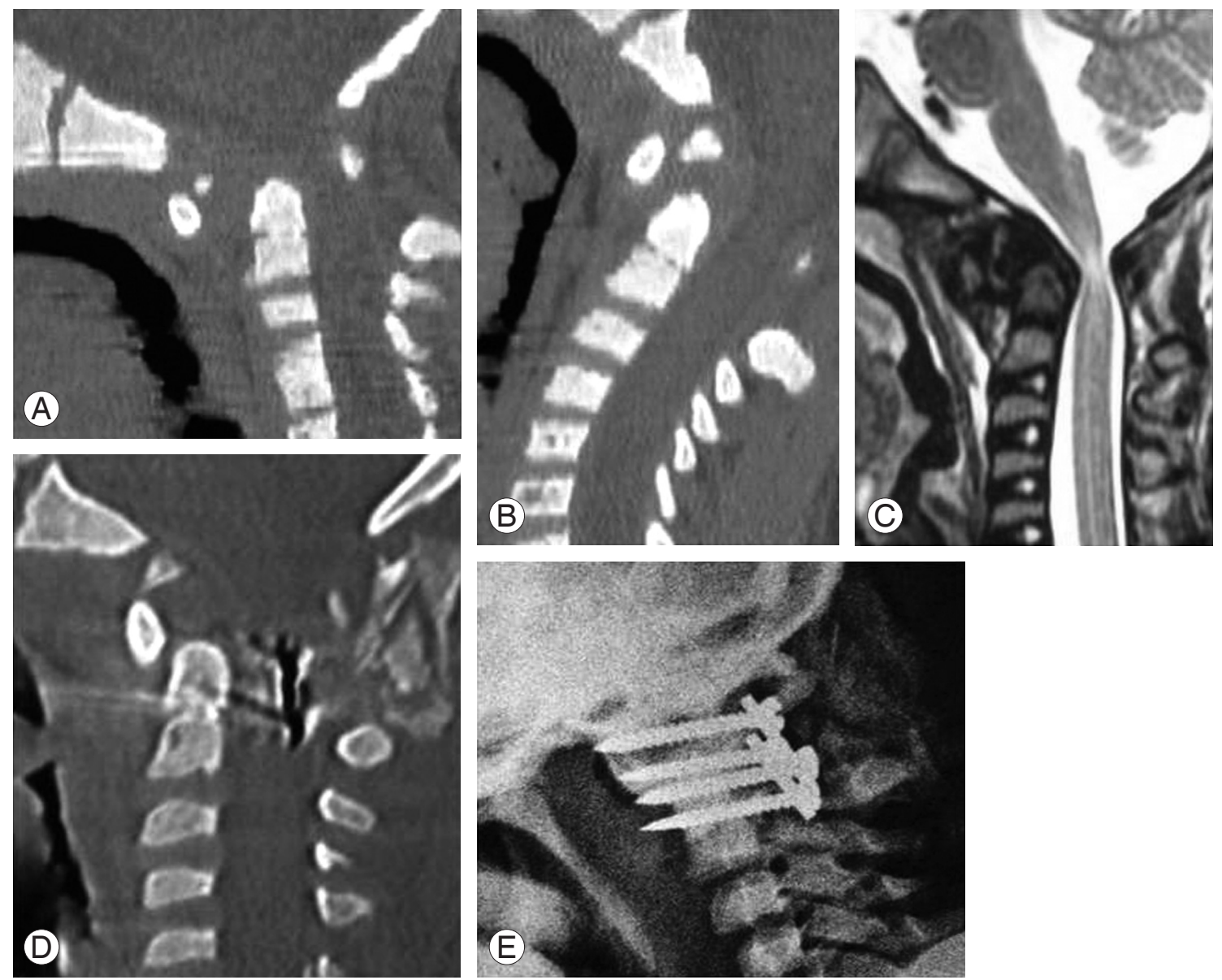

Fig. 1. Images of a 12-year-old male. (A) Computed tomography (CT) scan (sagittal view) with the head in flexed position shows severe atlantoaxial dislocation. There is os odontoideum. (B) CT scan with the head in extension shows reduction in the atlantoaxial dislocation. (C) T2-weighted magnetic resonance imaging shows severe cord compression. (D) Postoperative CT scan shows fixation with satisfactory alignment. (E) Lateral view of plain X-ray showing plate and screw fixation. 
during surgery. Neuronavigation systems can be helpful in this regard, but are not essential during surgery.

\section{Basilar Invagination (Figs. 2, 3)}

Chronic atlantoaxial instability leads to partially reducible or a 'fixed' atlantoaxial dislocation [13]. Basilar invagination is a type of or a manifestation of chronic or longstanding atlantoaxial instability. In long-standing instabilities musculoskeletal and neural malformations abound that are an attempt of the nature to tide over the crisis and reduce the effects of instability. In the year 2004 we suggested that atlantoaxial joint is seldom if at-all entirely fused or fixed and can be manually opened and realigned and fixed [14]. The concept that in cases with basilar invagination, atlantoaxial joint is unstable and not fixed and is manually distractable and reducible has radically changed the strategy of treatment for previously considered fixed or irreducible atlantoaxial dislocation $[13,14]$. The treatment protocol shifted towards 'craniovertebral realignment' and fixation-arthrodesis rather than decompression and bone removal.

In our publication in the year 2004, we had divided basilar invagination into two groups on the basis of presence (group A) or absence (group B) of clinical and radiological features of instability [14]. We had discussed the treatment option of craniovertebral realignment in cases with group A basilar invagination. Whilst we
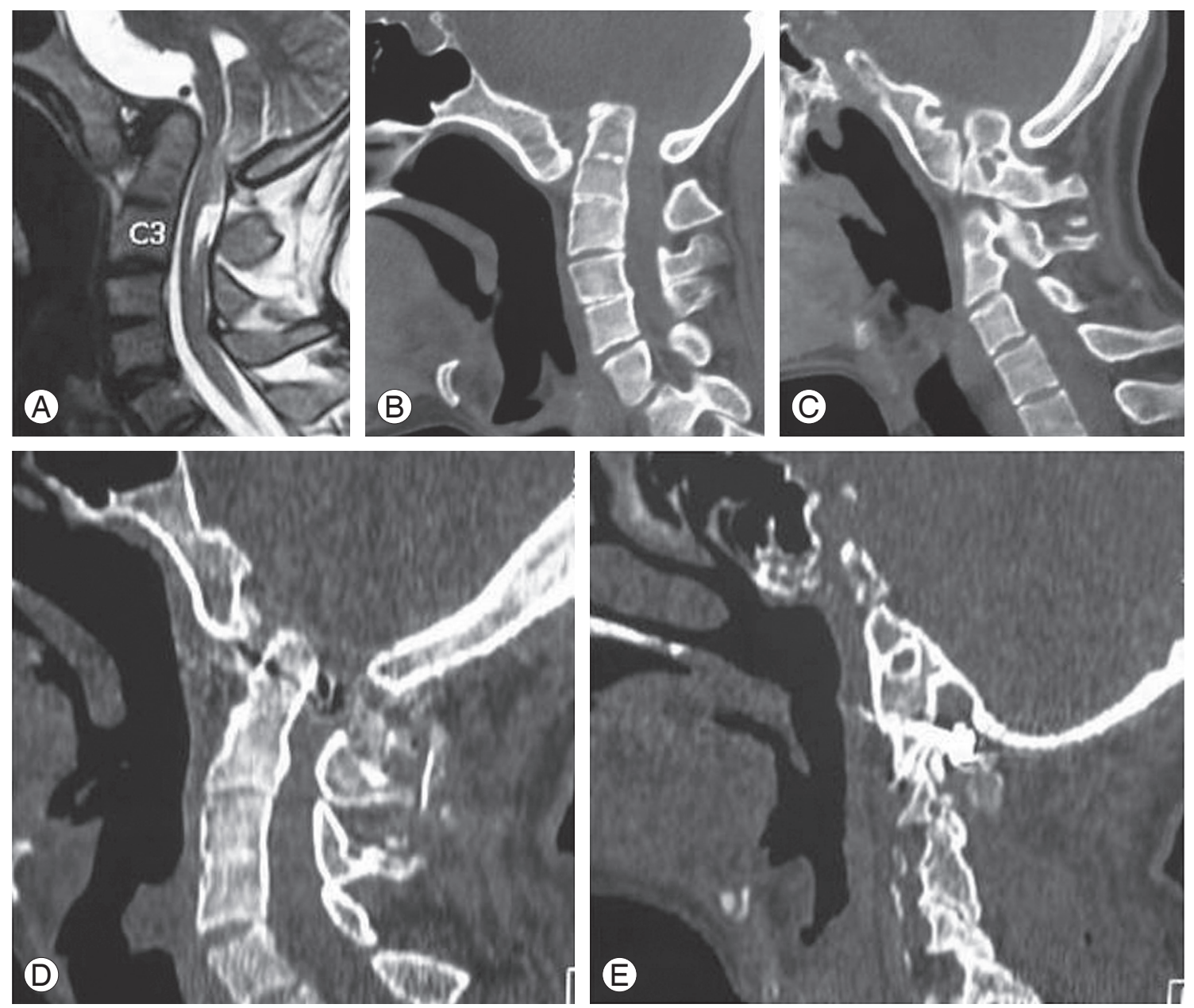

Fig. 2. Images of a 32-year-old male. (A) T2-weighted magnetic resonance imaging shows group A basilar invagination. Chiari malformation is observed. III-defined cord changes are observed in the spinal cord at C3 spinal level. (B) Computed tomography (CT) scan showing basilar invagination. Assimilation of atlas and C2-3 fusion can be observed. (C) Sagittal image with the section passing through the facets. Facets of $\mathrm{C} 1$ and $\mathrm{C} 2$ are in severe mal-alignment. (D) Postoperative image following surgery that involved distraction, reduction and fixation of the atlantoaxial joint. Please note the reduction of basilar invagination. (E) CT scan image showing realignment of the facets. 
suggested transoral decompression for group A basilar invagination, we suggested that for group B basilar invagination, foramen magnum decompression might be the best surgical option as we considered the atlantoaxial joint fixed in this subgroup of patients. However, as our understanding matures in the treatment of basilar invagination, we realize that basilar invagination of all varieties is a result of long standing atlantoaxial instability [15]. Atlantoaxial fixation is the treatment of basilar invagination and the most optimal form of treatment is by direct facetal fixation.

\section{Facetal Instability}

Assessing the atlantodental interval and its alterations on lateral profile radiological dynamic imaging has been the traditional method of diagnosing and monitoring atlantoaxial instability. Evaluating facetal alignments can be an additional method of evaluating instability. Computerized tomography scanning is a useful mode of investigation of
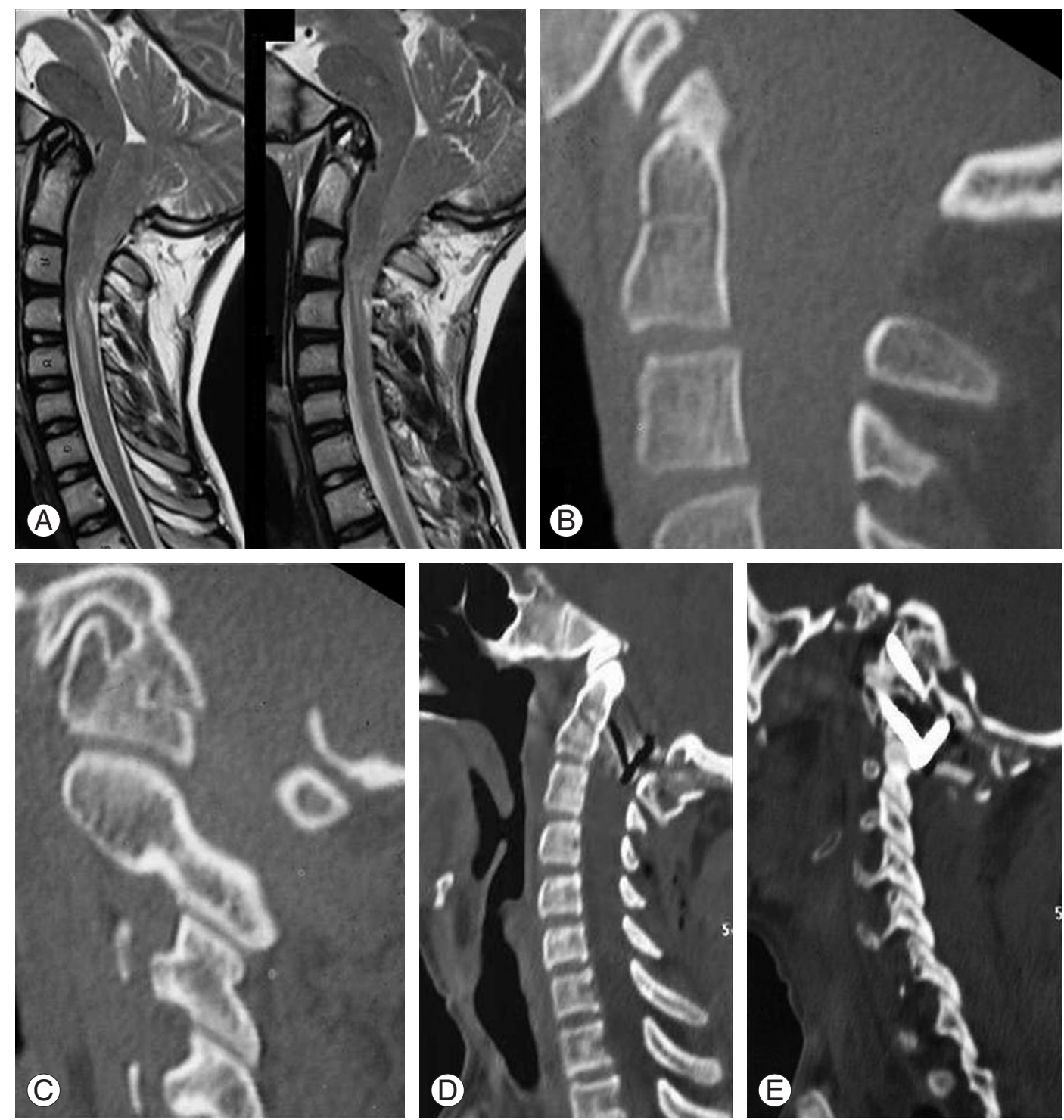

Fig. 3. Images of a 18-year-old male. (A) T2-weighted magnetic resonance imaging shows group B basilar invagination. There is Chiari malformation and syringomyelia. (B) Computed tomography (CT) scan showing basilar invagination. There is assimilation of atlas. (C) CT scan section through the facets showing that the facets are in alignment (type 3 instability). (D) Postoperative image showing stabilization. (E) CT section through the facets showing lateral mass plate and screw fixation. 
the craniovertebral junction in general and in evaluation of the status of facets in particular. The facets of atlantoaxial joint are large, rectangular in lateral profile and are placed one over the other like bricks. We identified three types of facetal malalignments [16,17]. Type 1 facetal malalignment was when the facet of atlas was dislocated anterior to the facet of axis on lateral imaging and simulates lumbosacral listhesis [18]. Such alignments are seen in cases with mobile and reducible atlantoaxial dislocations and more often in cases with group A basilar invagination. In this form of dislocation the odontoid process is displaced posteriorly and superiorly, resulting in atlantoaxial dislocation as manifested by an increase in atlantodental interval and basilar invagination. The odontoid process directly indents into the craniocervical cord and results in symptoms related to direct neural compression. The symptoms are relatively acute in such cases. Musculoskeletal alterations like short neck, torticollis, Klippel-Feil abnormalities, platybasia, secondary spondylotic changes and neural alterations like Chiari malformation and syringomyelia are relatively less frequently encountered in cases associated with type 1 atlantoaxial facetal instability. Type 2 facetal instability is when the facet of atlas is dislocated posterior to the facet of axis. Type 3 facetal instability is when the facets are in alignment and the instability is diagnosed on the basis of clinical and radiological evidences and more importantly it is diagnosed by direct surgical manipulations during surgery. In types 2 and 3 atlantoaxial facetal dislocations, atlantodental interval is not altered and the odontoid process does not directly indent into the neural structures. The neural deficits are delayed, symptoms are longstanding and are associated with multiple musculoskeletal and neural alterations. Types 2 and 3 facetal dislocations are more often associated with group B basilar invagination and cases with Chiari malformation and syringomyelia.

\section{Secondary Natural Protective Mechanisms in the Event of Chronic Atlantoaxial Instability}

Musculoskeletal changes associated with basilar invagination like short neck, torticollis, platybasia, KlippelFeil abnormalities, and spondylotic spinal changes are manifestations of chronic and subtle atlantoaxial instability $[19,20]$. The instability is more often of types 2 and 3 facetal dislocation. Following atlantoaxial fixation, all these secondary musculoskeletal alterations have a po- tential for reversal in the immediate postoperative phase $[19,20]$. This fact suggests that the musculoskeletal alterations are not congenital defects or a result of embryonic dysgenesis, but are a manifestation of chronic instability, and essentially have a protective function. These musculoskeletal abnormalities are not primary in nature or the cause of the problem, but are a secondary effect or result of the problem. On similar lines, we have recently identified that Chiari malformation and syringomyelia are also secondary or protective formations and are a result of long-standing atlantoaxial instability [21,22]. Chiari malformation can be simulated as nature's air bag that places it in position in the event of manifest or unidentified atlantoaxial instability. Like the air bag, the herniated tonsils act as a cushion that provides a protection to the neural structures and prevents pinching between bones. In the similar light, it appears that syringomyelia is also a protective self-neural destruction that works in the larger interest of the patient. It appears that the nature identifies the presence of instability and initiates a protective response much earlier than it is possible to recognize by alterations in the radiological parameters. The protective measures delay the onset of symptoms. The symptoms in such cases are subtle, long-standing and relentlessly progressive in nature. Presence of Chiari malformation and/ or syringomyelia are indicators of atlantoaxial instability. This is similar to the fact that short neck, torticollis and bone fusions are manifestation of atlantoaxial instability. The treatment in cases having basilar invagination (groups A and B) with/without Chiari malformation and with/without syringomyelia should be directed towards atlantoaxial stabilization and aimed at segmental arthrodesis (Fig. 4). Inclusion of the occipital bone in the fusion construct provides a suboptimal form of stabilization and can be counter-productive. Foramen magnum bone or dural decompression is not necessary. Although the patient might improve following foramen magnum decompression, the results are frequently only temporary. The improvement simulates relief to the affected car driver following deflating a full air bag. Essentially, foramen magnum decompression may temporarily assist in amelioration of symptoms, but in the long run is counterproductive.

As the understanding about craniovertebral junction issues grows, it is realized that majority of the pathogenetic issues are related to atlantoaxial instability. Stabilization of the atlantoaxial joint forms the treatment. The trend has 
changed over the years from decompression to segmental fixation. Both anterior tansoral and posterior foramen magnum decompressions are gradually slipping into the realm of history [23]. Operations that involve fixation of occipital bone and multiple spinal segments in the fixation construct are also getting less favor.
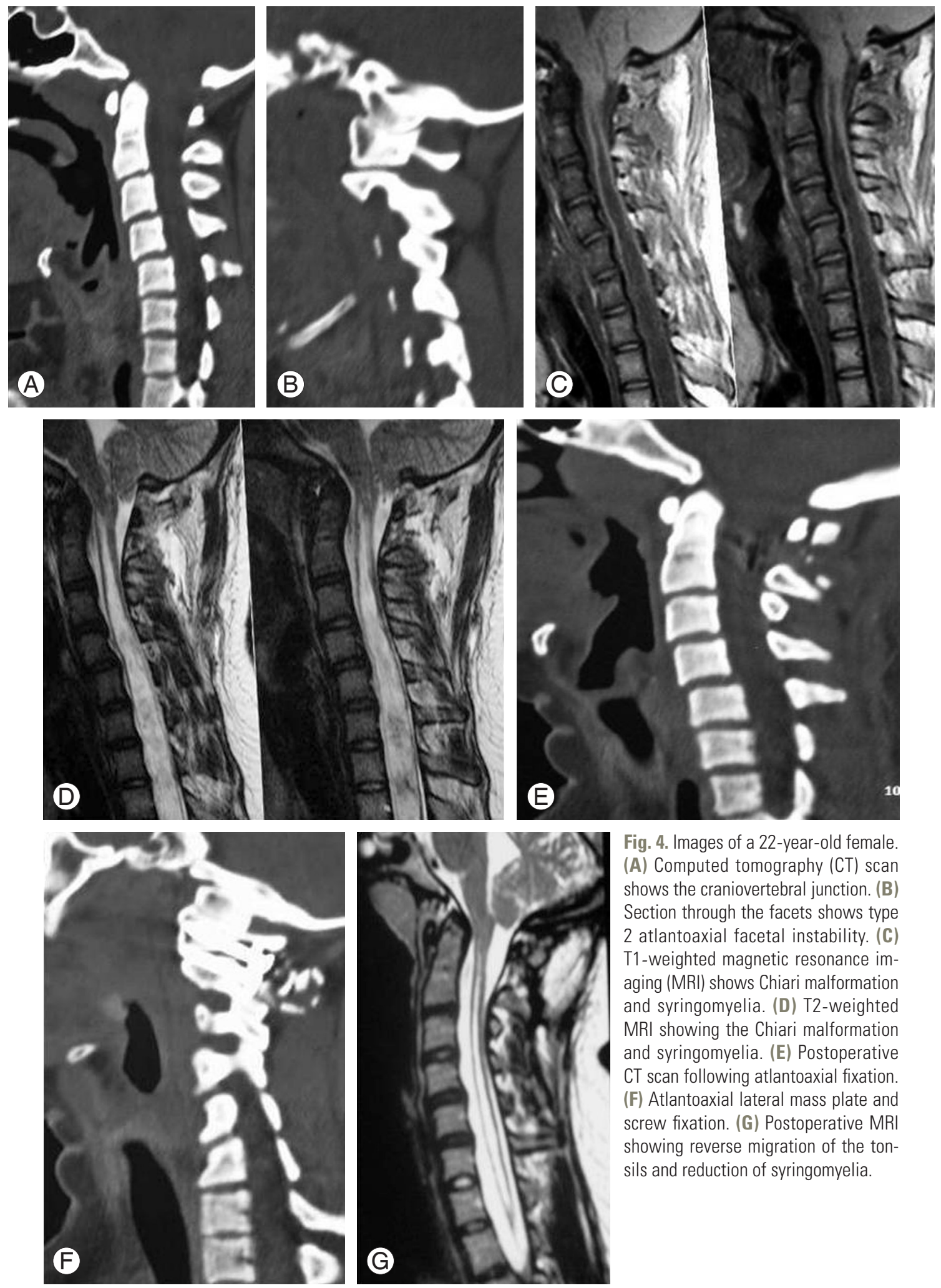

Fig. 4. Images of a 22-year-old female. (A) Computed tomography (CT) scan shows the craniovertebral junction. (B) Section through the facets shows type 2 atlantoaxial facetal instability. (C) T1-weighted magnetic resonance imaging (MRI) shows Chiari malformation and syringomyelia. (D) T2-weighted MRI showing the Chiari malformation and syringomyelia. (E) Postoperative CT scan following atlantoaxial fixation. (F) Atlantoaxial lateral mass plate and screw fixation. (G) Postoperative MRI showing reverse migration of the tonsils and reduction of syringomyelia. 


\section{Atlantoaxial Instability Associated with Cervical Spondylosis}

Craniovertebral abnormalities have frequently been associated with cervical spondylosis. In our earlier review, we hypothesized that features of cervical spondylosis and bone fusions are secondary spinal events related to primary instability at the atlantoaxial joint. In the years 2004 and 2008, we reported our experience with high cervical disc/ $s$ related cord compression and its association with basilar invagination and atlantoaxial instability and stressed upon the significance of primarily addressing the instability at craniovertebral junction $[24,25]$. We also identified that there is a potential for reversal of secondary spondylotic features following operations that aim at segmental atlantoaxial fixation and arthrodesis. It is therefore important to identify the presence of craniovertebral abnormalities when the patient is being investigated for cervical spondylosis. In general direct operation on cervical spine can be avoided in such cases and are not even be indicated.

Primary craniovertebral junction region degenerative changes and related instability has only rarely been reported [26]. However, recent studies have identified craniovertebral junction to be frequently involved with degenerative changes and is a common cause of cervical pain and myelopathy. Degenerative changes in the craniovertebral junction have been associated with reduction in atlantoaxial joint space, facetal instability, and osteophyte formation in and around the facets, apical ligament and other ligaments and in the retro-odontoid space [27]. Such degenerative changes suggest the possible presence of instability in the region that needs surgical treatment.

The craniovertebral segment of cervical spine is largely excluded or ignored from the umbrella of spinal spondylosis. We recently identified presence of atlantoaxial facetal instability in association with cervical subaxial single or multi-level degenerative spondylotic changes [26]. There was no evidence of spinal canal compromise or cord compression at the level of craniovertebral junction and dynamic imaging did not reveal any odontoid process related instability or cord compression. However, we identified type 2 atlantoaxial facetal instability in some such cases. Although no morphological studies are available that discuss alignment of the facets of atlas and axis during dynamic movements, it appears that some degree of facetal mal-alignment can be considered to be within the spectrum of normal variation. We have identified the association of types 2 and 3 facetal instability in cases with chronic or long-standing craniovertebral instability. Such types of instability are associated with group B basilar invagination and cases with Chiari malformation and syringomyelia. In essence, it appears that type B atlantoaxial facetal instability that is frequently the cause of basilar invagination can also be the cause or an association of cervical spondylosis. On the basis of this hypothesis, it appears that as atlantoaxial fixation forms the treatment of basilar invagination, it can also form the treatment of selected cases of cervical spondylosis.

On the basis of our experience, it appears that atlantoaxial facetal instability must be investigated in all cases of cervical spondylosis, particularly when the symptoms are disproportionate to the extent and degree of compression of the cervical cord related to spondylosis. High subaxial cervical spondylosis needs special focus on the atlantoaxial instability in general and facetal mal-alignment in particular. It needs to be assessed if only atlantoaxial stabilization will suffice in such cases. It is also necessary to assess if the instability of the spine is more generalized in cases with cervical spondylosis than what is observed on radiological imaging that focuses on status of neural structures [28]. More importantly, dynamic relationships of the facets needs to be evaluated on the basis of radiological and clinical studies based on normal population to evaluate the extent of facetal mal-alignment that could be considered within the range of normal variation.

\section{Conflict of Interest}

No potential conflict of interest relevant to this article was reported.

\section{References}

1. Goel A, Bhatjiwale M, Desai K. Basilar invagination: a study based on 190 surgically treated patients. J Neurosurg 1998;88:962-8.

2. Menezes AH. Primary craniovertebral anomalies and the hindbrain herniation syndrome (Chiari I): data base analysis. Pediatr Neurosurg 1995;23:260-9.

3. Gallie WE. Fractures and dislocations of the cervical spine. Am J Surg 1939;46:495-9.

4. Brooks AL, Jenkins EB. Atlanto-axial arthrodesis by the wedge compression method. J Bone Joint Surg Am 1978;60:279-84. 
5. Dickman CA, Sonntag VK, Papadopoulos SM, Hadley MN. The interspinous method of posterior atlantoaxial arthrodesis. J Neurosurg 1991;74:190-8.

6. Jeanneret B, Magerl F. Primary posterior fusion C1/2 in odontoid fractures: indications, technique, and results of transarticular screw fixation. J Spinal Disord 1992;5:464-75.

7. Goel A, Laheri V. Plate and screw fixation for atlantoaxial subluxation. Acta Neurochir (Wien) 1994;129: 47-53.

8. Goel A, Desai KI, Muzumdar DP. Atlantoaxial fixation using plate and screw method: a report of 160 treated patients. Neurosurgery 2002;51:1351-6.

9. Goel A. Cervical ganglion 2 (CG2) neurectomy: a window to the atlantoaxial joint. World Neurosurg 2012;78:78-9.

10. Gupta S, Goel A. Quantitative anatomy of the lateral masses of the atlas and axis vertebrae. Neurol India 2000;48:120-5.

11. Goel A. Double insurance atlantoaxial fixation. Surg Neurol 2007;67:135-9.

12. Cacciola F, Phalke U, Goel A. Vertebral artery in relationship to $\mathrm{C} 1-\mathrm{C} 2$ vertebrae: an anatomical study. Neurol India 2004;52:178-84.

13. Goel A, Kulkarni AG, Sharma P. Reduction of fixed atlantoaxial dislocation in 24 cases: technical note. J Neurosurg Spine 2005;2:505-9.

14. Goel A. Treatment of basilar invagination by atlantoaxial joint distraction and direct lateral mass fixation. J Neurosurg Spine 2004;1:281-6.

15. Goel A. Instability and basilar invagination. J Craniovertebr Junction Spine 2012;3:1-2.

16. Goel A. Goel's classification of atlantoaxial "facetal" dislocation. J Craniovertebr Junction Spine 2014;5:3-8.

17. Goel A. Facetal alignment: basis of an alternative Goel's classification of basilar invagination. J Craniovertebr Junction Spine 2014;5:59-64.

18. Kothari M, Goel A. Transatlantic Odonto-Occipital
Listhesis: the so-called basilar invagination. Neurol India 2007;55:6-7.

19. Goel A, Shah A. Reversal of longstanding musculoskeletal changes in basilar invagination after surgical decompression and stabilization. J Neurosurg Spine 2009;10:220-7.

20. Goel A, Nadkarni T, Shah A, Ramdasi R, Patni N. Bifid anterior and posterior arches of atlas: surgical implication and analysis of 70 cases. Neurosurgery 2015;77:296-306.

21. Goel A. Is Chiari malformation nature's protective "air-bag"? Is its presence diagnostic of atlantoaxial instability? J Craniovertebr Junction Spine 2014;5:1079.

22. Goel A. Is atlantoaxial instability the cause of Chiari malformation? Outcome analysis of 65 patients treated by atlantoaxial fixation. J Neurosurg Spine 2015;22:116-27.

23. Goel A. Can foramen magnum decompression surgery become historical? J Craniovertebr Junction Spine 2015;6:49-50.

24. Goel A. High cervical C3-4 'disc' compression associated with basilar invagination. Neurol India 2008;56:68-70.

25. Goel A, Phalke U, Cacciola F, Muzumdar D. Surgical management of high cervical disc prolapse associated with basilar invagination: two case reports. Neurol Med Chir (Tokyo) 2004;44:142-5.

26. Goel A. Posterior atlantoaxial 'facetal' instability associated with cervical spondylotic disease. J Craniovertebr Junction Spine 2015;6:51-5.

27. Goel A, Shah A, Gupta SR. Craniovertebral instability due to degenerative osteoarthritis of the atlantoaxial joints: analysis of the management of 108 cases. J Neurosurg Spine 2010;12:592-601.

28. Goel A. Vertical facetal instability: Is it the point of genesis of spinal spondylotic disease? J Craniovertebr Junction Spine 2015;6:47-8. 